\title{
CINTIGRAFIA OSEA TRIFASICA CON Tc-99m MDP EN EL DIAGNOSTICO Y MANEJO DE INFECCIONES OSTEOARTICULARES AGUDAS EN NIÑOS
}

\author{
Drs. Claudia Coll $C^{(1)}$, José Luis Cifras $V^{(2)}$, Teresa Massardo $V^{(1)}$, Hernán Moya $S^{(3)}$.
}

1. Medicina Nuclear, Departamento de Medicina, Hospital Clínico Universidad de Chile.

2. Servicio de Ortopedia y Traumatología Infantil Hospital Félix Bulnes C.

3. Servicio de Ortopedia y Traumatología Infantil Hospital Roberto del Río

\begin{abstract}
One of the most important causes of residual damage in skeleton is incompletely treated osteoarticular infections (OAI). Goal: to assess the value of emergency three-phase bone scan (3FBS) in the evaluation of OAI. Methods: Thirty-eight children were studied with 54 3FBS. Results: Global analysis: $76 \%$ of the cases were principally articular; staphylococcus germs and hip's affection were the common. Nine patients with preoperative scan were positive with 16 foci, 86\% confirmed by surgery cultivate. Seven children had negative 3FBS without posterior clinical symptomatology; in 5 of them it was demonstrated soft tissue involvement with normal osteoblastic phase. In 14/17 cases evaluated after surgical intervention, there was a relation between the scan and the clinical course. In two of those cases, in the 3FBS a new location was found.

Conclusion: 3FBS is a helpful tool in the diagnosis and assessment of acute $O A I$ in pediatric population. A positive $3 F B S$ is highly accurate for $O A I$ and a negative one rules it out securely.
\end{abstract}

Key words: Three-Phase bone scans, Ostearticular infection, Children.

Resumen: La principal causa de daño esquelético residual son las infecciones osteoarticulares (IOA) con un tratamiento inadecuado. Objetivo: Determinar el valor del cintigrama óseo trifásico (CO3F) de urgencia en la evaluación de IOA. Material y método: Se estudiaron 38 niños en quienes se efectuaron 54 CO3F.

Coll C. y cols. Cintigrafia ósea trifásica con Tc-99m MDP en el diagnóstico y manejo de infecciones osteoarticulares agudas en niños. Rev Chil Radiol 2002; 8:83-89.

Correspondencia: Dra. Claudia Coll $C$.

Hospital Clínico Universidad de Chile, Medicina Nuclear. Santos Dumont 999 1E. Independencia, Santiago.

Fono: (2) 7770569 - Fax: (2) 7777618

Email:ccoll@ns.hospital.uchile.cl
Resultados: Análisis Global: $76 \%$ de los casos fueron principalmente articulares; el germen más frecuente fue Staphylococcus y la articulación más afectada la cadera. Nueve pacientes con $\mathrm{CO} 3 \mathrm{~F}$ preoperatorio fueron positivos con 16 focos, $86 \%$ confirmados con cultivo quirúrgico. Siete niños tuvieron $\mathrm{CO} 3 \mathrm{~F}$ negativo sin sintomatología clínica posterior. En cinco casos el estudio fue negativo para compromiso osteoarticular, pero se demostró compromiso de partes blandas con fase osteoblástica normal. En 14 de 17 casos evaluados post-cirugía, hubo relación entre el CO3F y la evolución clínica, en dos de esos casos en el CO3F se encontró una nueva localización. Conclusión: El CO3F es una herramienta útil en el diagnóstico y evaluación de las IOA agudas en población pediátrica. Un CO3F positivo es altamente seguro de IOA y uno negativo lo descarta con certeza. Palabras Claves: Cintigrafía ósea trifásica, Infección ostearticular, Artritis Séptica,

Osteomielitis, Niños.

\section{Introducción}

Las infecciones osteoarticulares (IOA) no tratadas o inadecuadamente tratadas, son la principal causa de daño residual, que afecta al esqueleto en crecimiento. Ellas se pueden clasificar según: a) Tiempo de evolución; b) Localización: Artritis séptica (AS), osteomielitis (OM) y osteoartritis que se, observa principalmente en niños menores de 18 meses, por la presencia, de vasos transfisiarios (Figura 1); c) Mecanismo de producción: Hematógeno, por contigüidad o directo. d) Agente etiológico que varía según la edad, aún cuando el staphylococcus aureus es responsable en un 60 a $70 \%$ de los $\operatorname{casos}^{(1-3)}$.

El diagnóstico es clínico, con síntomas generales y locales como lo son el dolor, posición antiálgica, impotencia funcional, contractura muscular, edema local e imposibilidad para cargar peso ${ }^{(2)}$. En los exámenes de laboratorio, destacan, el hemograma con leucocitosis y desviación a izquierda; a veces 


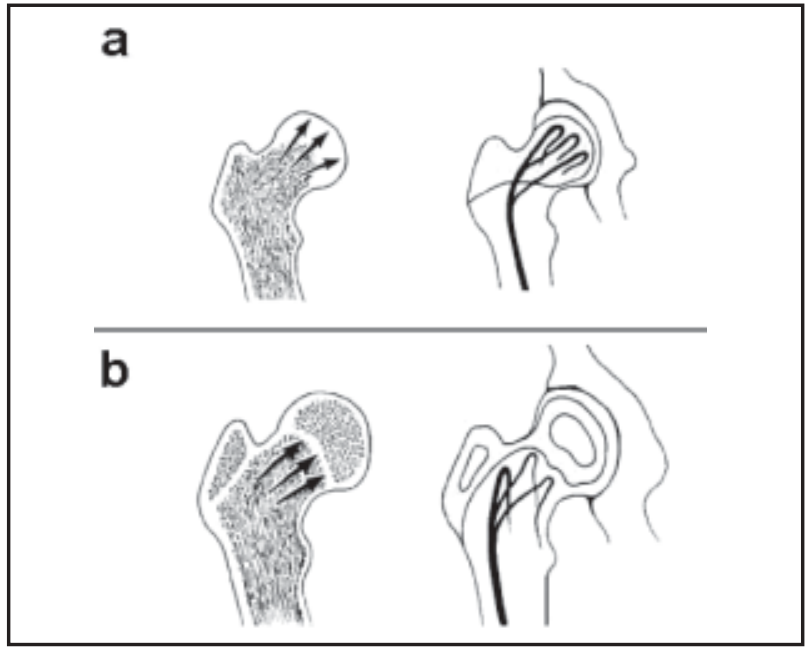

Figura 1 a,b. Flujo sanguíneo en fémur proximal e infección a. Neonato y lactante menor: Los vasos metafisiarios atraviesan la físis (flechas), de esta forma la infección afecta la epífisis e invade la articulación. b. Niños mayores: La físis (flechas) sirve como una barrera para "separar" las infecciones óseas de las articulares (modificado de referencia 1).

leucopenia; velocidad de sedimentación y proteína $C$ reactiva aumentadas. Los hemocultivos son positivos en un $50 \%$ de los $\operatorname{casos}^{(4-8)}$.

La radiología simple detecta tardíamente la enfermedad demostrando en AS: Aumento de amplitud del espacio articular, distensión de la cápsula, subluxación en caderas o deformidad de la superficie articular. En la OM aguda se puede observar: Aumento de partes blandas entre los 5 a 10 días, rarefacción ósea y reacción periostal después de los 7 a 12 días $^{(2)}$. La ultrasografía puede detectar precozmente derrame articular o subperióstico(9).

El cintigrama óseo ( $\mathrm{CO}$ ) es un estudio ampliamente disponible en los centros de medicina nuclear y su interpretación es rápida; permite un diagnóstico precoz de IOA, demostrando focos no sospechados clínicamente, tiene una alta sensibilidad, aunque una menor especificidad. Ambas aumentan al utilizarse las fases vasculares o $\mathrm{CO}$ trifásico $(\mathrm{CO} 3 \mathrm{~F})^{(10-20)}$.

Las ventajas del CO3F son ${ }^{(13,18,19,21,22)}$ : Alta sensibilidad que es cercana al $90 \%$, ya que disminuye los falsos positivos ${ }^{(20,23)}$, es de fácil disponibilidad en los centros de medicina nuclear, tiene un relativo bajo costo con relación a otros estudios imagenológicos, proporciona imágenes de cuerpo entero, útiles en cuadros sépticos con foco no precisado, su informe es rápido, siempre antes de 24 horas, usa baja dosis de radiación y no es operador dependiente.

La principal desventaja es su relativa baja especificidad.

\section{Infección osteoarticular: Fisiopatología}

Los gérmenes alcanzan la metáfisis a través de

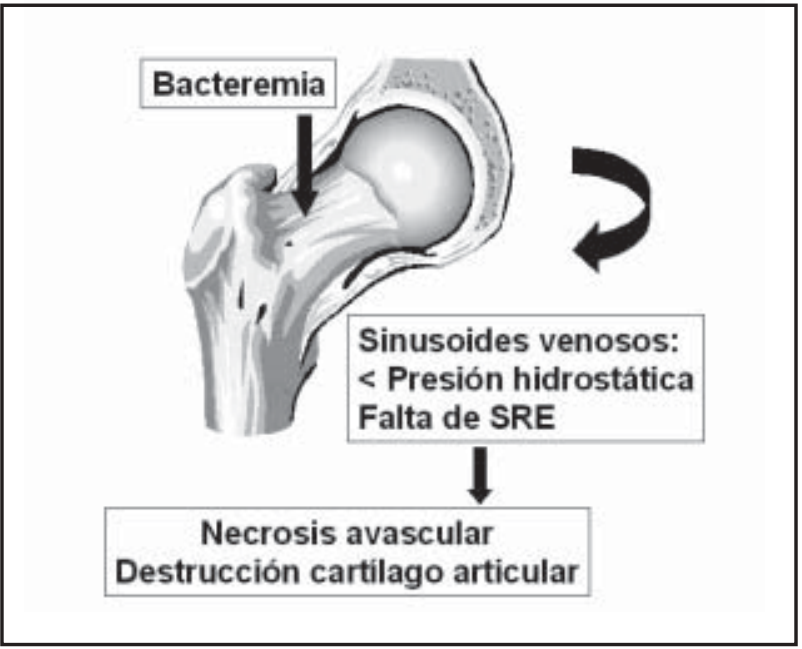

Figura 2. Fisiopatología de las Infecciones osteoarticulares.

la arteria nutricia ocupando los sinusoides venosos, donde se anidan, por una presión hidrostática muy baja y por ausencia de sistema retículo-endotelial. Posteriormente, se produce trombosis e infarto óseo, con necrosis avascular, existiendo daño óseo entre las 24 a 48 horas. A nivel articular existe hiperemia sinovial y edema, con aumento del líquido sinovial y producción de fibrina, que forma adherencias, habiendo destrucción del cartílago articular (Figura 2).

Los sitios más frecuentemente afectados son: rodilla, cadera, codo y tobillo en AS y las metáfisis proximal de la tibia, distal del fémur, proximal del húmero, distal de cúbito y radio en $\mathrm{OM}^{(1,2,24)}$.

Los radiofármacos ávidos por hueso son aquellos análogos del calcio, grupos hidroxilos o fosfatos. El de elección es el metilén difosfonato (MDP) marcado con Tc-99m, por su mayor tasa de absorción ósea y clearance sanguíneo (25-28). Su fijación se hace a nivel de la fase mineral, lo que depende de: la vascularización, la quimioabsorción, la captación mineral del hueso y enzimas.

\section{Pacientes y método}

El objetivo de este estudio fue determinar el valor del CO3F, de urgencia, en la evaluación de IOA agudas en niños, correlacionando los resultados cintigráficos con el seguimiento clínico y diagnóstico de egreso.

Entre los meses de Septiembre del 2000 y Febrero del 2002 se estudiaron prospectivamente 38 niños derivados a Medicina Nuclear por sospecha de IOA, realizándose en ellos $54 \mathrm{CO}$.

Criterios de inclusión fueron: 1) Alta sospecha clínica de IOA aguda y 2) Sospecha de localización secundaria de un proceso IOA conocido.

Se excluyeron pacientes con antecedentes de fractura antigua y sospecha de cuadros infeccioso subagudos o crónicos.

El promedio de edad fue de $4 \pm 5$ años (rango: 17 días a 18 años), $66 \%$ de los casos eran hombres. 
En 9 casos el CO se realizó previo a la intervención quirúrgica, con un total de 18 exámenes, considerando los controles de estos pacientes.

Otro grupo de 17 niños se estudiaron después de una cirugía por IOA previa, con el objetivo de pesquisar otros focos, el estudio se efectuó entre 1 a 34 días postoperatorios con un promedio de 5 días. En ellos se efectuaron 24 exámenes.

En un tercer grupo de 12 niños con sospecha clínica de IOA se efectuaron 12 estudios no seguidos de cirugía.

Se realizó seguimiento de todos los niños con un mínimo de 3 meses después del alta.

La cintigrafía ósea trifásica fue realizada según las normas establecidas en la Sección de Medicina Nuclear del Hospital Clínico de la Universidad de Chile ${ }^{(4,29,30)}$.

La dosis de Tc-99m MDP fue de 250 microCi por $\mathrm{Kg}$ de peso, con un mínimo de $1 \mathrm{mCi}$, administrada por inyección endovenosa. Se realizó estudio de 3 fases: una primera fase angiográfica, seguida de una fase de vascularización temprana o pool sanguíneo y una última fase ósea. En algunos casos se agregó una $4^{\circ}$ fase para mejorar la relación entre órgano blanco y fondo. En los pacientes en que no existía un foco claro de IOA se realizaron imágenes de cuerpo completo en fase de pool sanguíneo.

La fase ósea se efectuó, al menos 2 horas después de la inyección. Se adquirieron imágenes de cuerpo entero y segmentarias en niños poco colaboradores, con adquisiciones estáticas de la zona sospechada. Las imágenes de $4^{\circ}$ fase se realizaron 18 a 24 horas postinyección.

El equipo usado fue gamma-cámara General Electric StarCam 3200 XS, con matriz de 256 y colimador de alta resolución.

\section{Interpretación}

El cintigrama óseo fue interpretado sobre la base del atlas de Hahn ${ }^{(31)}$, según la edad y la simetría en las imágenes. Normalmente las articulaciones y vértebras presentan una leve mayor fijación del MDP y en los cartílagos de crecimiento existe una hiperfijación marcada del trazador.

Se consideró anormal cualquier alteración en la captación ósea o en tejidos blandos. La fijación ósea anormal se manifiesta como, hueso patológico metabólicamente activo (foco hipercaptante) o hipometabolismo óseo, manifestado como zona de hipocaptación del MDP o zona fría asociada o no a hipovascularización.

\section{Resultados}

Los resultados del cintigrama óseo fueron comparados con los hallazgos quirúrgicos, biopsia y cultivo de la lesión y con otros estudios de imágenes disponibles. El estándar de oro utilizado fue el diagnóstico al alta. El estudio es principalmente descriptivo, pero se determinó sensibilidad, especificidad, valores predictivos positivo y negativo y seguridad.

Análisis global: Se demostraron 33 focos de IOA en 26 niños que se distribuyeron en 18 focos de osteoartritis y 15 focos de osteomielitis. Estos últimos presente en 9 niños.

El germen más frecuente fue el staphylococcus aureus (40\%), seguido por el S. coagulasa (-) (35\%) y por el streptococcus (10\%). Según edad se identifico como germen más frecuente, S. aureus en 3 de 6 casos de niños menores de 1 mes, staphylococcus coagulasa negativo en 3 de 5 casos de lactantes y S. aureus en 5 de 15 pacientes pre-escolares y escolares (Tabla I).

\begin{tabular}{|c|c|c|c|}
\hline GERMEN & $\mathrm{RN}$ & Lactantes & $\begin{array}{c}\text { Pre-escolares } \\
\text { y Escolares }\end{array}$ \\
\hline S. Aureus & 3 & - & 5 \\
\hline Streptoccocus (SbHGA) & 1 & - & 1 \\
\hline S. coagulasa $(-)$ & 1 & 3 & 3 \\
\hline Serratia & 1 & - & - \\
\hline S. Pneumoniae & - & 1 & - \\
\hline Salmonella & - & - & 1 \\
\hline Cultivo negativo & - & 1 & 5 \\
\hline Total & 6 & 5 & 15 \\
\hline
\end{tabular}

La articulación más afectada fue la cadera comprometida en un $42 \%$ de los pacientes y la rodilla en un $14 \%$. En casos de OM el fémur fue el más afectado observándose compromiso en un $11 \%$ de los casos.

Otras localizaciones menos frecuentes fueron hombros en 3 casos, pie y metatarso en 3 casos, articulaciones sacroilíacas en 2 casos y un caso de tobillo, clavícula, codo y muñeca.

La sensibilidad global del estudio fue de $87,5 \%$, especificidad de $75 \%$, valores predictivos positivo y negativo de $87.5 \%$ y $75 \%$, respectivamente y una seguridad de $83,3 \%$.

Análisis por grupo de pacientes: Se analizan a continuación por separado los tres grupos de estudio Pacientes con estudio preoperatorio: En los 9 pacientes de este grupo el CO fue positivo y todos fueron intervenidos dentro de las 24 horas de efectuado el CO. Se demostraron 16 focos; 13 de ellos confirmados por cirugía, biopsia y/o cultivo de la lesión que constituyen un $81 \%$ de verdaderos positivos (Figuras 3 y 4 ), tres focos, en 2 pacientes, no tuvieron confirmación con un $19 \%$ de falsos positivos.

De los nueve pacientes hubo dos casos en que la 
sospecha clínica fue infección de la cadera. Sin embargo, en ambos el estudio cintigrafico demostró un foco en otro sitio de la extremidad inferior, los cuales fueron operados y confirmados.

No encontramos falsos negativos en este grupo cuando comparamos los resultados del CO3F con el diagnóstico de alta (Tabla II).

En un neonato, se encontró en el pie izquierdo, un área fotopénica tanto en las fases vasculares como en la ósea, la cirugía, demostró un absceso a ese nivel con compromiso óseo inicial, el cultivo fue positivo para serratia. Los $2 \mathrm{CO}$ de control realizados, al mes y dos meses del primero, no demostraron otros focos, existiendo normalización del área fotopénica en el control del mes.

En total se efectuaron 18 estudios en este grupo incluyendo el estudio inicial y los controles. En una paciente el control demostró nuevos focos de localización, los cuales fueron tratados médicamente como IOA.

De las 16 localizaciones, sólo en 12 se tuvo antecedente de cultivo tomado del foco, de los cuales $7(58 \%)$ resultaron positivos.

Otros estudios imagenológicos, estaban descritos sólo en seis casos en la ficha clínica (radiografías, ultrasonografías y en un caso resonancia magnética) de ellos sólo en uno las imágenes fueron negativas en presencia de un cintigrama óseo positivo.

El resultado de la biopsia tomada durante la cirugía estaba presente en 6 casos, de ellos en 5 era concordante con patología infecciosa.

Pacientes con estudio post-operatorio: En 14 de 17 casos (82\%) evaluados posterior a cirugía se observó correlación entre el estudio isotópico y la evolución clínica.

En dos de los 14 pacientes el cintigrama fue negativo en la localización inicial, pero fueron encontrados nuevos focos infecciosos, en uno de ellos la evolución clínica no confirmó el foco. En el otro caso se reintervino el foco inicial, con hallazgos concordantes con patología séptica (Tabla II).

De los 24 exámenes realizados en este grupo se detectaron 17 focos de los cuales en 16 hubo correlación con el diagnóstico de alta. En siete focos el estudio isotópico fue negativo, sin embargo, en cuatro se demostró la existencia de IOA.

Pacientes estudiados y no operados: De los doce niños en este grupo, siete tuvieron un CO normal y no fueron intervenidos. En el seguimiento a los tres meses no presentaron sintomatología clínica osteoarticular (verdaderos negativos). Uno de estos pacientes tenía una ultrasonografía de caderas alterada, el estudio de CO3F y seguimiento fueron negativos para IOA. (Tabla II).

De los 12 pacientes en 5 casos el CO3F demostró, en las fases vasculares, compromiso de tejidos blandos ( 3 celulitis, 1 flebitis y 1 caso de parálisis braquial derecha en recuperación), con fase osteoblástica normal. Ellos fueron tratados médicamente (Figura 5).

\section{Discusión}

Dado que los signos de IOA se manifiestan tardíamente en la radiografía simple ${ }^{(21)}$, es necesario contar con un examen que pueda diagnosticar en forma precoz, segura y con un bajo costo este tipo de afecciones. La ultrasonografía, en presencia de clínica compatible, puede orientar al diagnóstico de la AS al demostrar derrame articular y subperiostico, pero es operador dependiente ${ }^{(9)}$. La RM, tiene una buena resolución anatómica, alta sensibilidad y especificidad, pero tiene un alto costo, poca disponibilidad en hospitales públicos y requiere, en niños, muchas veces anestesia general, además es órgano específico, lo que limita su uso en la búsqueda de foco desconocido.

El staphylococus aureus tuvo en nuestro estudio una frecuencia de $40 \%$ y al igual que lo publicado fue el germen más frecuente ${ }^{(1,2)}$.

En la mayoría de las series clínicas ${ }^{(2)}$, la AS afecta la articulación de la rodilla y la OM la metáfisis proximal de la tibia, en nuestra experiencia, sin embargo la articulación de la cadera y la metáfisis distal del fémur, fueron las más afectadas. Dado que estas localizaciones presentan una mayor dificultad diagnóstica, requieren un mayor apoyo de imágenes.

Cuando la sospecha clínica de IOA es alta, el CO es innecesario y debe reservarse para casos

Tabla II. Valores estadísticos de la $\mathrm{CO} 3 \mathrm{~F}$ realizada por grupo de pacientes.

\section{GRUPO DE}

PACIENTES

Sensibilidad (\%)

Especificidad (\%)

VPP* $(\%)$

VPN* ${ }^{*}(\%)$

Seguridad (\%)
Preoperatorio

100

NE

80

100

87.5
Post-Cirugía

81.0

75.0

94.4

42.9

80.0
No Operado

$\mathrm{NE}^{*}$

100

NE

100

100
GLOBAL

87.5

75.0

87.5

75.0

83.3

*VPP: Valor predictivo positivo; VPN: Valor predictivo negativo; NE: No evaluable, por escaso número. 

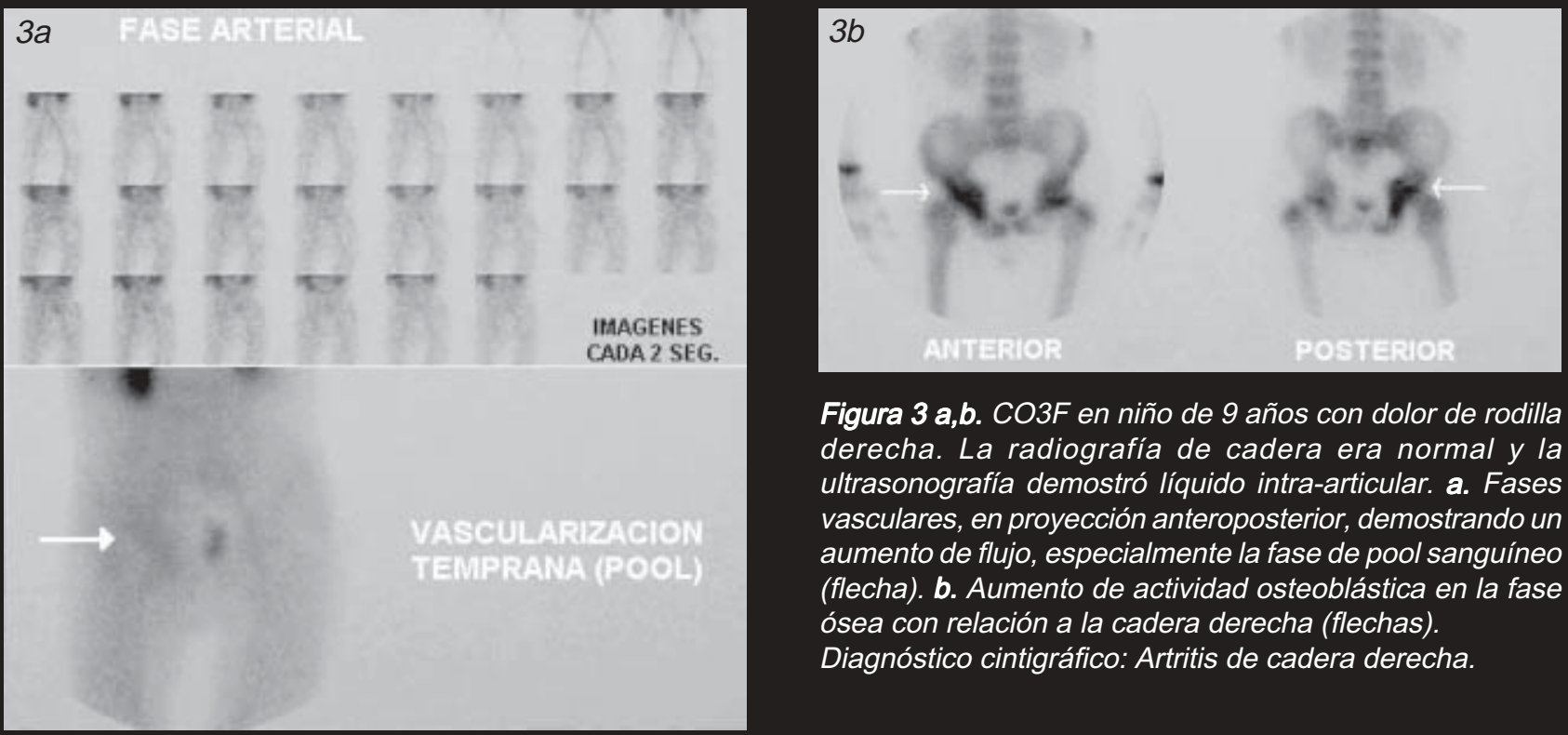

Figura 3 a,b. CO3F en niño de 9 años con dolor de rodilla derecha. La radiografía de cadera era normal y la ultrasonografía demostró líquido intra-articular. a. Fases vasculares, en proyección anteroposterior, demostrando un aumento de flujo, especialmente la fase de pool sanguíneo (flecha). b. Aumento de actividad osteoblástica en la fase ósea con relación a la cadera derecha (flechas).

Diagnóstico cintigráfico: Artritis de cadera derecha.
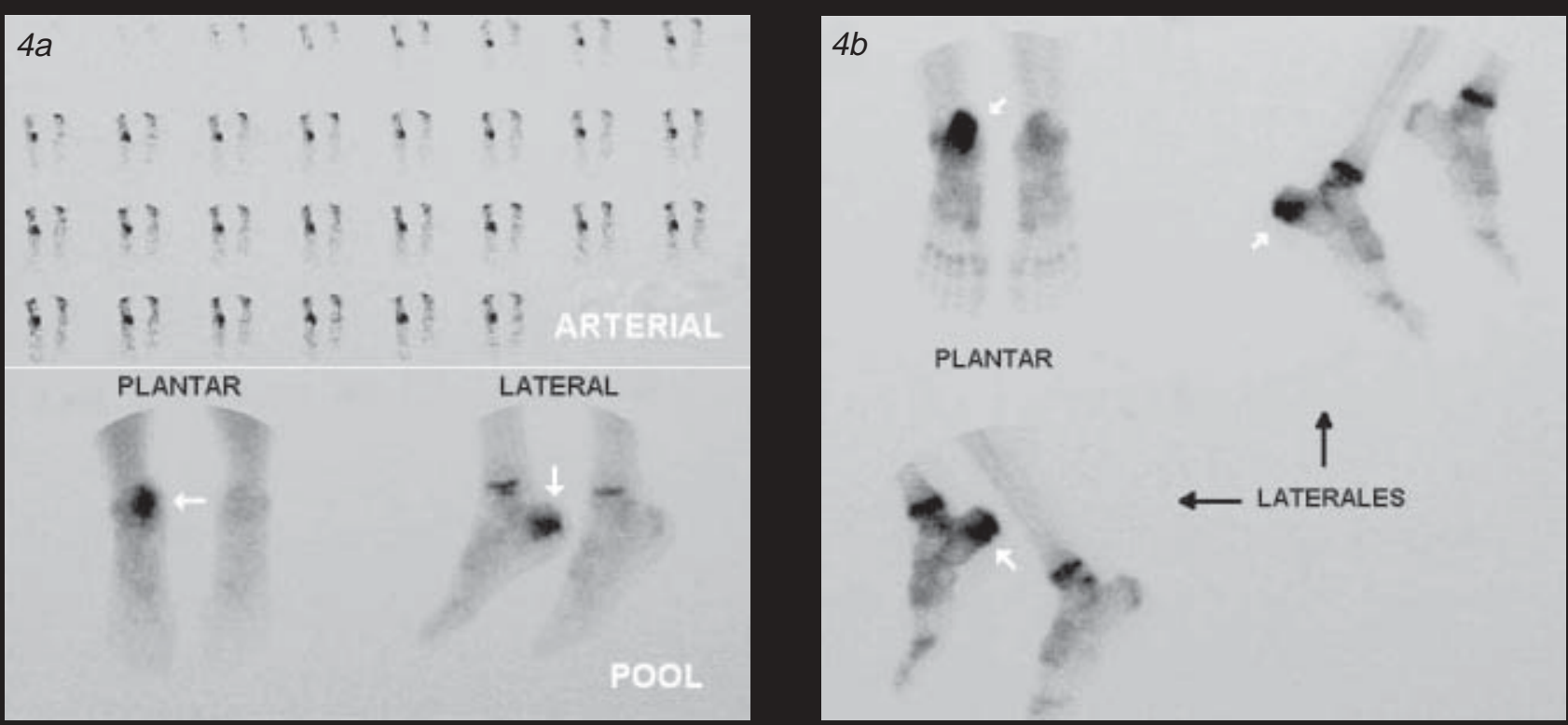

Figura 4 a,b. CO3F de niño de 13 años, que consulta por dolor en pie izquierdo. Radiografía normal. a. Fases vasculares demostrando un aumento significativo en el flujo (flechas) b Fase ósea con aumento del metabolismo óseo (flechas blancas). Diagnóstico: Osteomielitis de calcáneo.

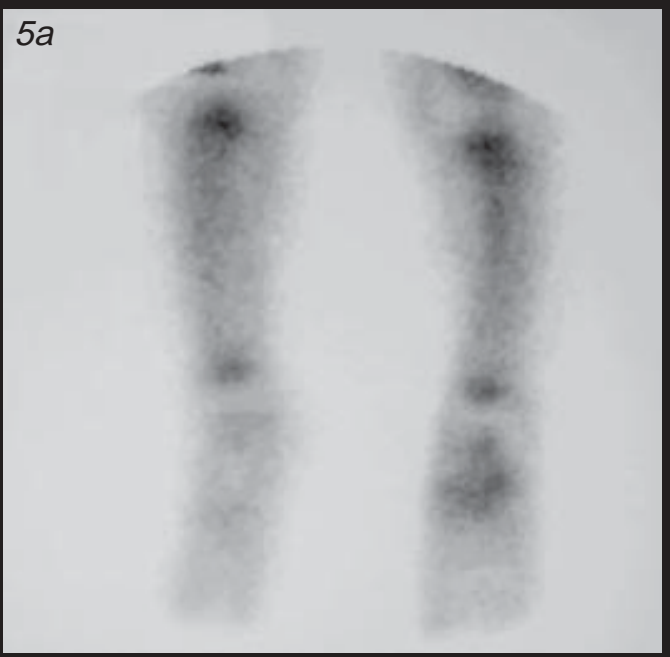

Figura 5. a,b. Cintigrama óseo. A. Fase vascular en proyección AP de piernas demuestra aumento difuso de flujo a nivel de la extremidad izquierda. b. Fase ósea normal. Imagen distal de fémures y proximal de tibias. 
complejos, en donde la clínica es poco evidente o existe sospecha de otros focos.

Desde la introducción en 1971 del uso de compuestos de fosfatos marcados con tecnecio para las imágenes del esqueleto ${ }^{(32)}$, la cintigrafía ósea, ha tenido excelentes reportes de seguridad diagnóstica en IOA variando de 56 a 100\% ${ }^{(25)}$. En nuestro estudio ella fue de $83 \%$.

En 1975 Gilday ${ }^{(18)}$ describió por primera vez las características cintigráficas para la AS y la OM. En la AS existe un aumento periarticular de la actividad osteoblástica. Este hecho no fue siempre tan evidente en nuestro trabajo debido al alto numero de menores de 18 meses quienes presentan procesos osteoartríticos por la presencia de vasos transfisiarios.

La capacidad de diferenciar clínicamente una celulitis de una OM no es siempre fácil, el CO3F permite un diagnóstico diferencial entre estas dos patologías ${ }^{(23)}$ lo que fue fácilmente demostrado en nuestro trabajo.

Se han descrito la presencia de focos fotopénicos en IOA estudiados con CO3F, su fisiopatología aún no está bien definida, se piensa que podría corresponder a isquemia secundaria a aumento de presión intraósea y subperióstica, en infección de gran virulencia y son visibles precozmente, generalmente dentro de los primeros 4 días. La teoría más aceptada es, sin embargo, una trombosis medular primaria causada por compresión de la microcirculación en el canal medular por abscesos intraóseos o presión extrínseca de la arteria nutricia por secreción purulenta subperiostal(13,15,25,33-36). Uno de nuestros pacientes presentó un área fotopénica en las 3 fases lo que representa un 2,6\%, menor a lo reportado por Pennington que fue de un $8,6 \%{ }^{(15)}$.

Aigner ${ }^{(22)}$ reportó una sensibilidad de $90 \%$ para la $\mathrm{CO} F$ en el diagnóstico de focos infecciosos en neonatos, similar a lo publicado por Maurer ${ }^{(20)}$ en población general (92\%). Nuestra sensibilidad global fue $88 \%$, y de un $100 \%$ en el grupo con diagnóstico preoperatorio. Esta sensibilidad disminuye en el grupo con estudio post-cirugía, probablemente debido al uso de antibióticos y a la terapia quirúrgica efectiva de las lesiones. Tuson ${ }^{(25)}$ refiere que los falsos negativos aumentan con el uso prolongado de terapia antibiótica previa al estudio cintigráfico, aun cuando también ello podría deberse a la transición de actividad osteoblástica que se produce entre la fotopenia y un foco hipercaptante.

Gilday ${ }^{(18)}$ y Maurer ${ }^{(20)}$ reportan un aumento significativo de la especificidad, al usar las fases vasculares por disminución de los falsos positivos, asociados a hiperemia ósea secundaria a infección de tejidos blandos contiguos. Una mayor disminución de falsos positivos ocurre al efectuar una $4^{\text {ta }}$ fase ${ }^{(37)}$. La especifidad global obtenida en nuestro estudio fue de $75 \%$, la que fue especialmente baja en estudios postoperatorios, principalmente por la patología inflamatoria inmediata post-cirugía.

Tuson ${ }^{(25)}$ separa los valores predictivos positivos para CO "caliente" versus "frío", el cual varía de un 82 a un $100 \%$. En nuestro estudio, al existir sólo un caso con área fotopénica se analizó globalmente, demostrando un valor de $88 \%$.

En nuestra serie el valor predictivo negativo fue mayor en los casos en que el estudio isotópico se efectuó previo a cirugía (100\%), con una disminución importante en el estudio postoperatorio (43\%), lo que está dado por un mayor número de falsos negativos.

La literatura reciente es escasa en el estudio del $\mathrm{CO}$, probablemente por ser una técnica ampliamente aceptada como útil y segura en el diagnóstico de patología infecciosa osteoarticular.

Concordamos con Aigner ${ }^{(22)}$ en que el cintigrama óseo trifásico, de urgencia, puede cambiar la conducta terapéutica, lo que tiene un buen rendimiento costo versus beneficio, además de implicancias en las secuelas que puede presentar un paciente joven.

\section{Conclusiones}

El cintigrama óseo trifásico es una herramienta útil en el diagnóstico y evaluación de las infecciones osteoarticulares agudas en la población pediátrica, especialmente en los estados precoces.

Un CO3F positivo es altamente probable de infección OA y un estudio negativo lo descarta con excelente seguridad.

\section{Bibliografía}

1. Dormans J, Drummond D. Pediatric hematogenous osteomyelitis: New trends in presentation, diagnosis, and treatment. J Am Acad Orthop Surg 1994; 2:333-341

2. Sucato D, Schwend R, Gillespie R. Septic arthritis of the hip in children. J Am Acad Orthop Surg 1997; 5:249-260

3. Wandl-Vergesslich KA, Breitenseher M, Fotter R. Diagnostic imaging in osteomyelitis. Characteristics in childhood. Radiology 1996; 36:805-812

4. Peltola I, Vahvanen V, Aalto K. Fever, C-reactive protein and erythrocyte sedimentation rate and monitoring recovery from septic arthritis: A preliminary study. J Pediatr Orthop 1984; 4: 170-174

5. Scott RJ, Christofersen MR, Robertson WW Jr y col. Acute osteomielitis in children: A review of 116 cases. J Pediatr Orthop 1990; 10:649-652

6. Unkila-Kallio L, Kallio MJT, Eskola J y col. Serum Creactive protein, erythrocyte sedimentation rate and white cell count in acute hematogenous osteomyelitis of children. Pediatrics 1994; 93:59-62

7. Morrisy RT. Bone and joint infections, en Morrisy RT (ed): Lovell and Winter's Pediatrics Orthopedics. $3^{\text {rd }}$ ed. Philadelphia; Lippincott, 1990, vol 1, pp 539-561 
8. Nade S. Acute septic artritis in infancy and childhood. J Bone Joint Surg (Br) 1983; 65: 234-241.

9. Zieger M, Dorr U, Schulz RD. Ultrasonography of hip joint effusions. Skeletal Radiol 1987; 16:607-611.

10. Berkowits ID, Wenzel W. Normal technetium bone scan in patients with acute osteomyelitis. Am J Dis Child 1980; 134:828-30.

11. Handmaker $\mathrm{H}$, Leonards $\mathrm{R}$. The bone scan in inflammatory osseous disease. Semin Nucl Med 1976; 6:95-105.

12. Scoles PV, Hilty MD, Sfakianakis GN. Bone scan patterns in acute osteomyelitis. Clin Orthop 1980; 153: 210-217.

13. Sullivan JA, Vasileff T, Leonard JC. An evaluation of nuclear scaning in orthopedics infections. J Pediatr Orthop 1981; 1: 73-9.

14. Majd M. Radionucleotide imaging in early detection of childhood osteomyelitis and its differentiation from cellulitis and bone infarction. J Ann Radiol 1997; 20;9-18.

15. Pennigton W. Photopenic bone scan osteomyelitis: A Clinical Perspective. J Pediatr Orthop 1999; 19: 695-698.

16. Canale ST, Harknes RM, Thomas PA y col. Does prior aspiration of bone and joints affect results of later bone scanning? J Pediatr Orthop 1985; 5:23-26.

17. Gilday DL. Problems in the scintigraphic detection of osteomyelitis. Radiology 1980; 135:791.

18. Gilday DL, Paul DJ, Paterson J. Diagnosis of osteomyelitis in children by combined blood pool and bone imaging. Radiology 1975; 117:331-5.

19. Aronson J, Garvin K, Seibert J y col. Efficiency of the bone scan for occult limping toddlers. J Pediatr Orthop 1992; 12:38-44.

20. Maurer AH, Chen DC, Camargo EE y col. Utility of threephase skeletal scintigraphy in suspected osteomyelitis: Concise communication. J Nucl Med 1981; 22:941-9.

21. Sammak B, Abd El, Bagi M y col. Osteomyelitis: a review of currently used imaging techniques. Eur Radiol 1999; 9:894-900.

22. Aigner RM, Fueger GF, Ritter G. Results of three-phase bone scintigraphy and radiography in 20 cases of neonatal osteomyelitis. Nucl Med Commun 1996; $17: 20-8$.
23. Kim EE, Haynie TP, Podoloff DA y col. Radionuclide imaging in the evaluation of osteomyelitis and septic arthritis. Crit Rev Diagn Imaging 1989; 29:257-305.

24. Green NE, Edwars K. Bone and joint infections in children. Orthop Clin North Am 1987; 18:555-576.

25. Tuson CE, Hoffman EB, Mann MD. Isotope bone scaning for acute osteomyelitis and septic arthritis in children. J Bone Joint Surg (Br) 1994; 76:306-310.

26. McCoy JR, Morissey RT, Seibert J. Clinical experience with a technetium scan in children. Clin Orthop 1981; 154: 175-180.

27 Peltola $\mathrm{H}$, Vahvanen V. A comparative study of osteomyelitis and purulent arthrirtis with special reference to etiology and rocovery. Infection 1984; 12: $75-79$.

28. O'Connor MK, Brown ML, Hung JC, Hayostek RJ. The Art of Bone Scintigraphy - Technical Aspects. J Nucl Med 1991; 32: 2332-2341.

29. Brown ML, O'Connor MK, Hung JC, Hayostek RJ. Technical aspects of bone scintigraphy. Radiol Clin North Am 1993; 31:721-30.

30. Donohoe K, Henkin E, Royal H, y col. Society of Nuclear Medicine Procedure Guideline for Bone Scintigraphy 2.01999.

31. Hahn K, Fischer S, Gordon I. Atlas of Bone Scintigraphy in the Developing Pediatric Skeleton. The normal skeleton, variants and pitfalls. Ed: Springer-Verlang. Berlin Heidelberg. Germany 1993: 7-272.

32. Subramanian G, McAfee JG. A new complex of Tc-99m for skeletal imaging. Radiology 1971; 99:192-6.

33. Jones DC, Cady RB. Cold bone scans in acute osteomielitis. J Bone Joint Surg 1981; 63-B:376-8.

34. Sfakianakis GN, Scoles $P$, Welch $M$ y col. Evolution of bone imaging findings in osteomyelitis in children. $\mathrm{J}$ Nucl Med 1978; 19: 706.

35. Sy MW, Westring DW, Weinberger G. Cold lesions on bone imaging. J Nucl Med 1975; 16:1013-6.

36. Williamson $\mathrm{Br}$, Sisrom CL. Femoral and acetabular photopenia associated with septic hip arthritis. Clin Nucl Med 1991; 16:52.

37. Israel O, Gips S, Jerushalmi J y col. Osteomyelitis and soft-tissue infection: differential diagnosis with 24 hour/ 4 hour ratio of Tc-99m MDP uptake. Radiology 1987; 163: 725-6. 